\title{
1 Sex-specific crossover rates did not change with parental age in
}

2 Arabidopsis

4 Ramswaroop Saini ${ }^{1,4}$, Amit Kumar Singh ${ }^{2}$, Geoffrey J. Hyde ${ }^{3}$ and Ramamurthy Baskar ${ }^{\text {* }}$

$7 \quad{ }^{1}$ Department of Biotechnology, Indian Institute of Technology-Madras, Bhupat and Jyoti Mehta

8 School of Biosciences, Chennai 600 036, India.

$9{ }^{2}$ School of Plant Sciences and Food Security, Tel Aviv University, Tel Aviv 6997801, Israel.

$10 \quad{ }^{3}$ Independent Researcher, Randwick, New South Wales, Australia

$11{ }^{4}$ Department of Biotechnology, Kalinga University, Raipur, Chhattishgarh 492101 India.

12

14 Running Title: Parental age did not influence sex-specific crossover rates in Arabidopsis

15

16 Key Words: Arabidopsis thaliana, meiotic recombination, parental age, centromere, crossing

17 over, heterochiasmy

19 Corresponding author: Ramamurthy Baskar

20 Email id $\quad$ : $\quad$ rbaskar@iitm.ac.in

21 Phone number : 044-2257-4110 
22 Address: Department of Biotechnology, Indian Institute of Technology-Madras, Bhupat and

23 Jyoti Mehta School of Biosciences, Chennai 600 036, India.

25 Abstract

27 Crossing over, the exchange of DNA between the chromosomes during meiosis, contributes

28 significantly to genetic variation. The rate of crossovers $(\mathrm{CO})$ varies depending upon the taxon,

29 population, age, external conditions, and also, sometimes, between the sexes, a phenomenon

30 called heterochiasmy. In the model plant Arabidopsis thaliana, the male rate of crossovers

31 (mCO) is typically nearly double the female rate (fCO). With increasing parental age, it has been

32 reported that the disparity decreases, because fCO rises while mCO remains stable. That finding,

33 however, is based on chromosome-based averaging, and it is unclear whether all parts of the

34 genome respond similarly. We addressed this point by examining how the level of heterochiasmy

35 responded to parental age in eight genomic intervals distributed across the five chromosomes of

36 Arabidopsis. Unlike the previous work, in each of the eight intervals, the level of heterochiasmy

37 did not change with age, that is, the ratio mCO:fCO remained stable. As expected, though,

38 amongst the intervals, the levels of heterochiasmy at any of the four ages examined, did vary.

39 We propose that while the levels of heterochiasmy in Arabidopis might decrease with age on a

40 chromosomal basis, as reported earlier, this is not true for all locations within each chromosome.

41 This has practical implications for plant breeding research, a major aim of which is identifying

42 ways to induce local increases in $\mathrm{CO}$ rates. 


\section{Introduction}

During meiotic crossing over, homologous chromosomes align and exchange paternally and maternally derived DNA. Crossovers $(\mathrm{CO})$ are one of the main sources of variation in sexually reproducing organisms, and as such, the rate at which they occur has considerable evolutionary significance (Ritz et al. 2017; Stapley et al. 2017). If the rate is too low, the organism has less chance of adaptation, if too high, an already effective genotype runs the risk of disruption. While

51 the rate of crossovers can vary across taxa, populations, and between and within individuals, the

52 possible scale of variation across these various levels appears remarkably constrained (Ritz et al.

53 2017). Nevertheless, the scope for some degree of $\mathrm{CO}$ rate variation exists for individual

54 organisms, and is of practical importance, both medically and economically. For example, the

55 frequencies of several forms of human chromosomal number abnormalities (in particular,

56 trisomies) correlate with the increased frequency of CO (Hussin et al. 2011; Alves et al. 2017).

57 In plant breeding, the development of 'elite' genotypes depends on meiotic COs that allow the

58 accumulation of desirable traits, and much research is focused on finding ways to increase local

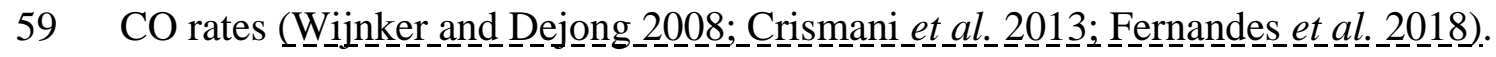

60 Interestingly, it is often not just the overall rate of $\mathrm{CO}$ that is important, but also the ratio of the

61 male and female rates of $\mathrm{CO}$ (henceforth, $\mathrm{mCO}$ and $\mathrm{fCO}$ ). In many taxa, these two rates differ to

62 a greater or lesser extent, a phenomenon called heterochiasmy (Ritz et al. 2017; Stapley et al.

63 2017). At the most extreme, in what is called achiasmy, one sex does not form chiasmata at all

64 (John et al. 2016; Satomura et al. 2019). This is the case,_for example, in the male of Drosophila,

65 in which chromosome alignment employs an alternative to the synaptonemal complex (McKee et 
al. 2012). In true heterochiasmy, the ratio between the rates of the more and less recombinative sexes can vary from 1.035 to 14 (Ritz et al. 2017). Evidence indicates that, for a truly

68 heterochiasmatic species, the sex that has the lower rate of $\mathrm{CO}$ will be the one for which genetic

69 stability in the haploid phase is most likely to be critical to the future organism's fitness

70 (Lenormand 2003; Lenormand and Dutheil 2005; Stapley et al. 2017). In Arabidopsis, for

71 example, its high self-pollination rate (95\%; (Charlesworth and Vekemans 2005) suggests that

72 the female haploid phase is most critical, thus possibly explaining why fCO has the lesser value

73 (Lenormand 2003; Lenormand and Dutheil 2005). The ratio of mCO:fCO in young Arabidopsis

74 seedlings is typically about 1.8 (Toyota et al. 2011; Giraut et al. 2011), but amongst different

75 accessions, the values can vary by about 22\% (López et al. 2012).

77 As well as having evolutionary drivers, both the overall, and sex-specific, CO rates, and also

78 mCO:fCO, are influenced by age and extrinsic stressors such as temperature, pathogens,

79 chemical exposure, and lack of nutrients (Hayman and Parsons 1962; Francis et al. 2007; Toyota

80 et al. 2011; Hussin et al. 2011; Martin et al. 2015; Halldorsson et al. 2016; Li et al. 2017;

81 Modliszewski and Copenhaver 2017; Saini et al. 2017; Stapley et al. 2017). The effect of age on

82 mCO:fCO, and its mechanistic basis, has been much studied in humans because the increased

83 rates of $\mathrm{CO}$ implicated in the chromosomal number abnormalities mentioned above mostly occur

84 in older women (Hussin et al. 2011; Chiang et al. 2012; Nagaoka et al. 2012; Alves et al. 2017).

86 For plant $\mathrm{CO}$, much less is known about age $\mathrm{x}$ sex interactions. For example, with respect to the

87 influence of age on patterns of heterochiasmy in Arabidopsis, there has only been one study

88 (Toyota et al. 2011); other studies have examined the response of mCO only (Francis et al. 2007; 
89 Li et al. 2017). In Toyota et al. (2011), the extent of heterochiasmy in primary shoots decreased

90 with age, because, although there was no change in $\mathrm{mCO}$, there was an increase in fCO. It is

91 unclear, however, whether this pattern is true for each location in the chromosome. That study

92 looked at 343 markers across the five chromosomes of the species, and reported on the average

93 change of $\mathrm{mCO}$ and $\mathrm{fCO}$ for each chromosome, taking the mean of rates for the set of each

94 chromosome's applicable markers. The likelihood of intrachromosomal variation of

95 heterochiasmic values is suggested by the results of Li et al. (2017). That study found that while

$96 \mathrm{mCO}$ in primary shoots did not significantly change with age for markers in five of nine genomic

97 intervals (thus in agreement with the earlier results of Toyota et al. 2011), the rates did

98 significantly increase in two intervals. The possibility of intrachromosomal variation in

99 Arabidopsis heterochiasmy is also supported by other studies that have shown that, at least at one

100 time point, the chromosomal average, and location-specific, values of mCO:fCO vary greatly

101 depending on which chromosome is examined, and the location with the chromosome \{Drouaud

102 et al., 2007; Giraut et al., 2011\}.

103

104 In this study we explore the possibility of intrachromosomal variation further, by looking at the

105 influence of parental age on $\mathrm{mCO}$, fCO, and $\mathrm{mCO}: \mathrm{fCO}$, using eight markers that cover all five

106 chromosomes of Arabidopsis. Plants were sampled at four time points that cover the full

107 reproductive duration of the Arabidopsis main shoot. We find that, while, at any one age, the

108 ratio mCO:fCO differed both inter- and intra-chromosomally, the ratio, and also mCO and fCO,

109 did not change with parental age of the main shoot. We believe the most likely reasons for the

110 apparent discrepancy between our results and previous findings (i.e. Toyota et al., 2011) is that:

111 (1) on the one hand, our small set of markers did not include any of the locations that exhibit an 
112 age-response by fCO nor, for that matter, any of those that exhibit an age-response by mCO (as

113 reported in $\mathrm{Li}, 2017)$; (2) on the other hand, by reporting only on the chromosomal averages of

114 mCO:fCO, the earlier study could not detect any of an intrachromosomal spectrum of age-

115 responses. It appears this spectrum is wide enough to include a lack of response at some

116 locations, as we have reported for all three parameters, and as Li et al. (2017) report for mCO.

\section{Materials and methods}

\section{Plant growth conditions}

120 Freshly harvested Arabidopsis seeds from Columbia or detector lines (described below) were

121 surface sterilized with $70 \%$ ethanol, followed by $0.5 \%$ bleach treatment for 3 min. Subsequently,

122 the seeds were washed thrice with sterile water and plated on autoclaved Murashige and Skoog

123 media (MS, with 3\% sucrose), pH 5.7, containing 0.05\% Plant Preservative Mixture (Biogenuix

124 Medsystem Pvt. Ltd., New Delhi, India) and incubated at $4^{\circ} \mathrm{C}$ in dark conditions, for

125 synchronized germination. After $48 \mathrm{~h}$, the plates were shifted to a seed germination chamber,

126 with a uniform light intensity of 8000 lux units (16-h light/8-h dark cycle). The temperature of

127 the chamber (Percival CU-36L6) was maintained at $22^{\circ} \mathrm{C}$ with a constant humidity of $80 \%$.

128 Three-week old seedlings were transferred from MS plates to soil and grown inside a plant 129 growth chamber (Percival AR-36L3). The soil had equal proportions of garden soil, peat, perlite, 130 and vermiculite (Keltech Energies Ltd., Bangalore, India). 


\section{Arabidopsis detector lines used to score CO rates}

133 To score CO rates, eight different detector lines covering at least one marker in each of the five

134 chromosomes were used. The detector lines Col3-4/20, 3158 and 3162 were kind gifts from

135 Avraham A. Levy (Department of Plant Sciences, Weizmann Institute of Science, Israel),

136 (Melamed-Bessudo et al. 2005). Another set of detectors, the traffic lines CTL1.2, CTL1.18,

137 CTL2.4, CTL4.7 and CTL5.17 were obtained from the Arabidopsis Biological Resource Center

138 (Ohio State University, USA), (Wu et al. 2015) (Table 1). In all the lines, the eGFP and dsRed

139 markers are driven by a seed-specific napin promoter. The detector lines, homozygous for both

140 markers were crossed with Columbia plants, and the seeds obtained (heterozygous for both eGFP

141 and dsRed) were used in the subsequent experiments.

143 Investigating parental age effect on $\mathrm{CO}$ rates

144 To examine the influence of parental age on CO rates, plants of the detector lines and Columbia

145 plants, of four different ages (40, 45, 50 and 55 DAS (days after sowing), were emasculated $48 \mathrm{~h}$

146 before pollination and reciprocally crossed with each other. Different colored threads were used

147 to mark emasculated and pollinated flowers of different age groups. For each age, approximately

14820 to 30 crosses were performed in three independent replicates. To score recombination during

149 megaspore formation (fCO), we used emasculated flowers from the detector lines and crossed

150 them with pollen from Columbia plants. Similarly, to estimate recombination rates during

151 microspore formation (mCO), we used a detector line as the pollen donor for emasculated 152 flowers of Columbia. 
155 The segregation of eGFP and dsRed markers (an indication of CO rates during micro- or mega-

156 sporogenesis in the detector line parent), was analysed by the manual counting of seeds. Seeds

157 were placed on a glass slide and analyzed under a Nikon Stereozoom Microscope (SMZ 1000)

158 equipped with filters specific for both eGFP and dsRed. Images were captured for eGFP and

159 dsRed separately and then both the images merged to identify the recombinant and non-

160 recombinant seeds. CO rates were estimated based on the segregation of eGFP and dsRed

161 markers. Of the four types of seeds obtained, seeds that fluoresce only either red or only green

162 were counted as having undergone a $\mathrm{CO}$, while the seeds that fluoresce for both red and green as

163 well as those that do not fluoresce at all, were counted as not having undergone a CO. MR rates

164 were calculated based on the formula:

165

166

$$
(\mathrm{R}+\mathrm{G})
$$

$$
(\mathrm{R}+\mathrm{G}+\mathrm{RG}+\mathrm{NFS})
$$

170 R- dsRed-only expressing seeds; G- eGFP only expressing seeds; RG- Seeds expressing both

171 dsRed and eGFP; NFS- non-fluorescent seeds. The 'rate' is actually more correctly called a

172 frequency, but we have used the term 'rate' because of its common usage in the literature. 


\section{Statistical analysis}

175 Meiotic $\mathrm{CO}$ rates follow a normal distribution and hence, a Gaussian generalized linear model

176 (GLM) with identity link function was used (Nelder and Wedderburn 1972). The linear

177 predictors were either the different ages, or the sex, of the detector-line parent. In all GLMs, the

178 data from groups were compared. Correction for multiple testing was done to maintain the

179 family-wise error rate at 5\% (Gabriel 1969). Therefore, the $P$ values were adjusted with a single-

180 step method that considered the joint multivariate $t$ distribution of the individual test statistic

181 (Bretz et al. 2016). The results were reported with the two-sided $P$ values adjusted for multiple

182 comparisons (Singh et al. 2015). All statistical analyses were carried out in R (Team 2014). To

183 adjust the $P$ values for multiple testing, the $\mathrm{R}$ package multcomp was used with the test

184 specification ‘single-step'(Bretz et al. 2016). Graphs were produced using GraphPad Prism 8.

186 Results

Heterochiasmy in eight intervals of Arabidopsis was unaffected by parental age

189 Using a set of eight Arabidopsis detector lines, we examined the influence of parental age on

190 male and female CO rates. The eight detector lines heterozygous for both eGFP/dsRed were

191 reciprocally crossed with Columbia wild type plants, with both parents being one of four ages

192 (40, 45, 50, and 55 DAS) and MR rates were examined in the collected seeds (Figure 1). The

193 eight intervals were distributed across all five chromosomes, and varied in length and the degree

194 of overlap with sub-telomeric or pericentomeric regions (Figure 2; Table 1). One interval (in line

195 CTL1.2) spanned the centromere. 
$\mathbf{A}$

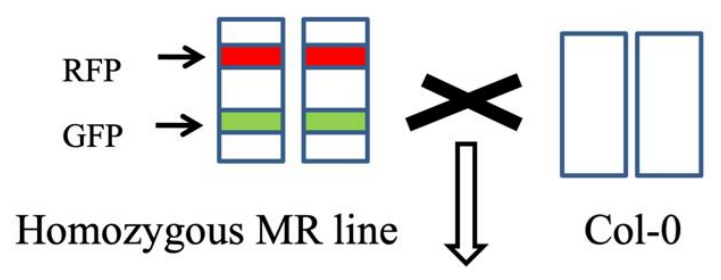

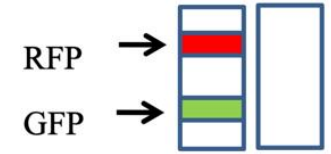

Heterozygous MR line

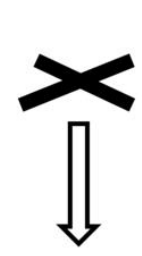

Reciprocal cross between MR detector lines and Columbia at different age group (40, 45, 50, and 55 DAS ).
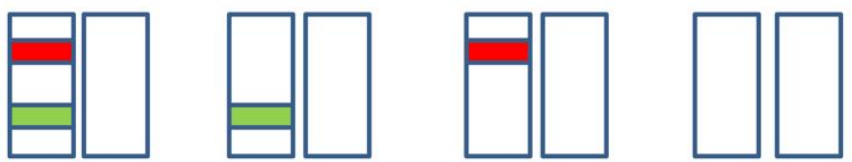

Products of meiotic recombination
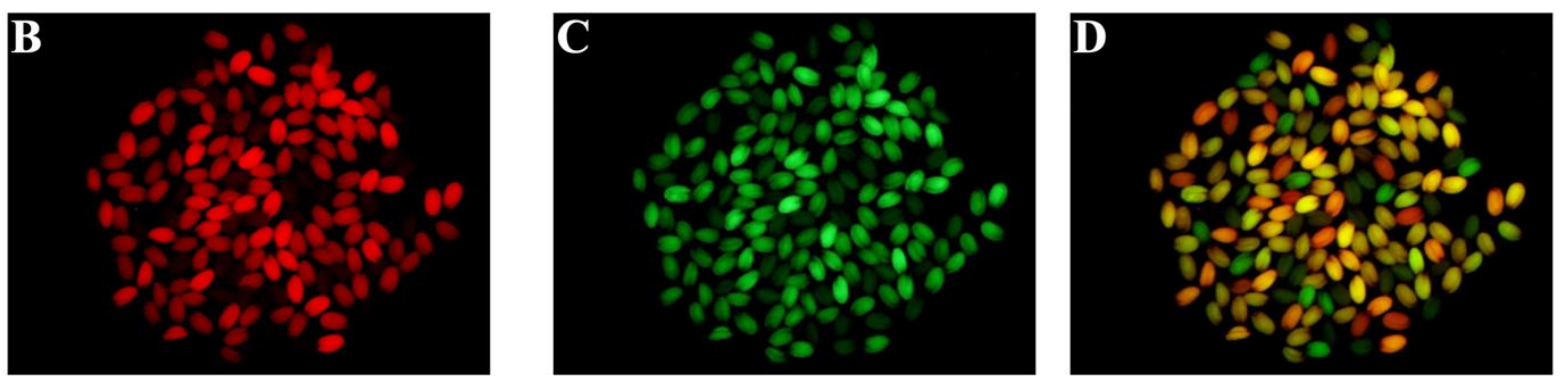

198 Figure 1 (A) A reciprocal cross between a heterozygous detector line and a Columbia plant

199 results in seeds with one of four fluorescence patterns: a blend of red and green; only green; only

200 red; no fluorescence. (B) A sample of seeds observed using a dsRed filter; (C) The same sample

201 of seeds observed using an eGFP filter. (D) Merged image of B and C showing the four different

202 patterns of fluorescence. Seeds in which a CO has occurred are those that either have only green

203 or only red fluorescence, as determined by manual assessment of one image type or (if

204 necessary) all three image types. 


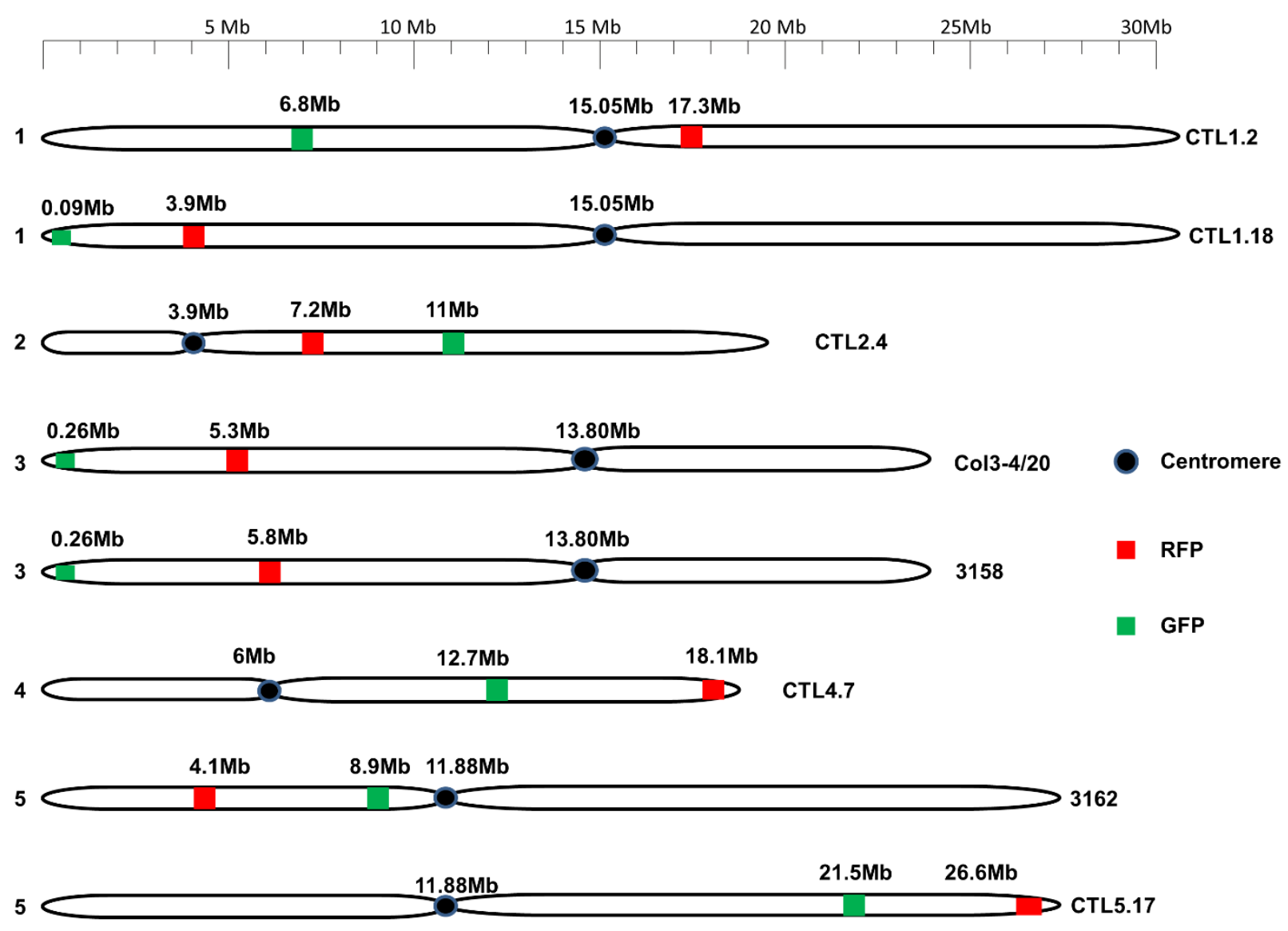

207 Figure 1. Physical maps of the chromosomes showing the location of the inter-marker

208 intervals in the detector lines tested. Positions of eGFP and dsRed were drawn on the physical

209 map using the chromosome map tool of The Arabidopsis Information Resource (TAIR). 


\section{Table 1 Structural features of the eight intervals used in this study}

\begin{tabular}{|c|c|c|c|c|c|}
\hline Line Name & Chr. No & $\begin{array}{l}\text { Centromere } \\
\text { position (Mb) }\end{array}$ & $\begin{array}{l}\text { Percentage of } \\
\text { interval that is } \\
\text { close to a } \\
\text { telomere* }\end{array}$ & $\begin{array}{l}\text { Percentage of } \\
\text { interval that is } \\
\text { close to a } \\
\text { centromere** }\end{array}$ & $\begin{array}{l}\text { Length of interval } \\
\text { (distance between } \\
\text { markers, } \mathrm{Mb} \text { ) }\end{array}$ \\
\hline $\begin{array}{l}\text { CTL1.2 } \\
\text { (Centromere is } \\
\text { between } \\
\text { markers) }\end{array}$ & 1 & 15.05 & 0 & 65 & 10.50 \\
\hline CTL1.18 & 1 & 15.05 & 100 & 0 & 3.81 \\
\hline CTL2.4 & 2 & 3.9 & 0 & 0 & 3.3 \\
\hline Col3-4/20 & 3 & 13.8 & 65 & 0 & 5.04 \\
\hline 3158 & 3 & 13.8 & 65 & 0 & 5.54 \\
\hline CTL4.7 & 4 & 6 & 43 & 0 & 5.4 \\
\hline 3162 & 5 & 11.88 & 0 & 22 & 4.8 \\
\hline CTL5.17 & 5 & 11.88 & 72 & 0 & 5.1 \\
\hline
\end{tabular}

216 The table summarises some key features of the intervals bounded by each pair of markers. *This percentage

217 was calculated by determining how much of the interval's length occurs within the first $15 \%$ or last $15 \%$ of the

218 chromosome's length. **This percentage was calculated by determining how much of the interval's length

219 occurred in the two regions that are on either side of the centromere (each region being $15 \%$ of the

220 chromosome's length). For both cases, the choice of $15 \%$ was based on the authors' visual analysis of the

221 distribution of male and female CO hotspots in each of the five chromosomes, as depicted in Figure 4, Giraut

222 et al. (2011).

224 For each of the eight intervals, there was no significant change in the ratio, $\mathrm{mCO}: \mathrm{fCO}$, as the

225 age of the male and female parents was increased (Figure 3). Neither did the two individual rates

226 that are used to calculate the ratio (i.e. mCO; and fCO) vary with age (Figure 3; Tables S1-8). 

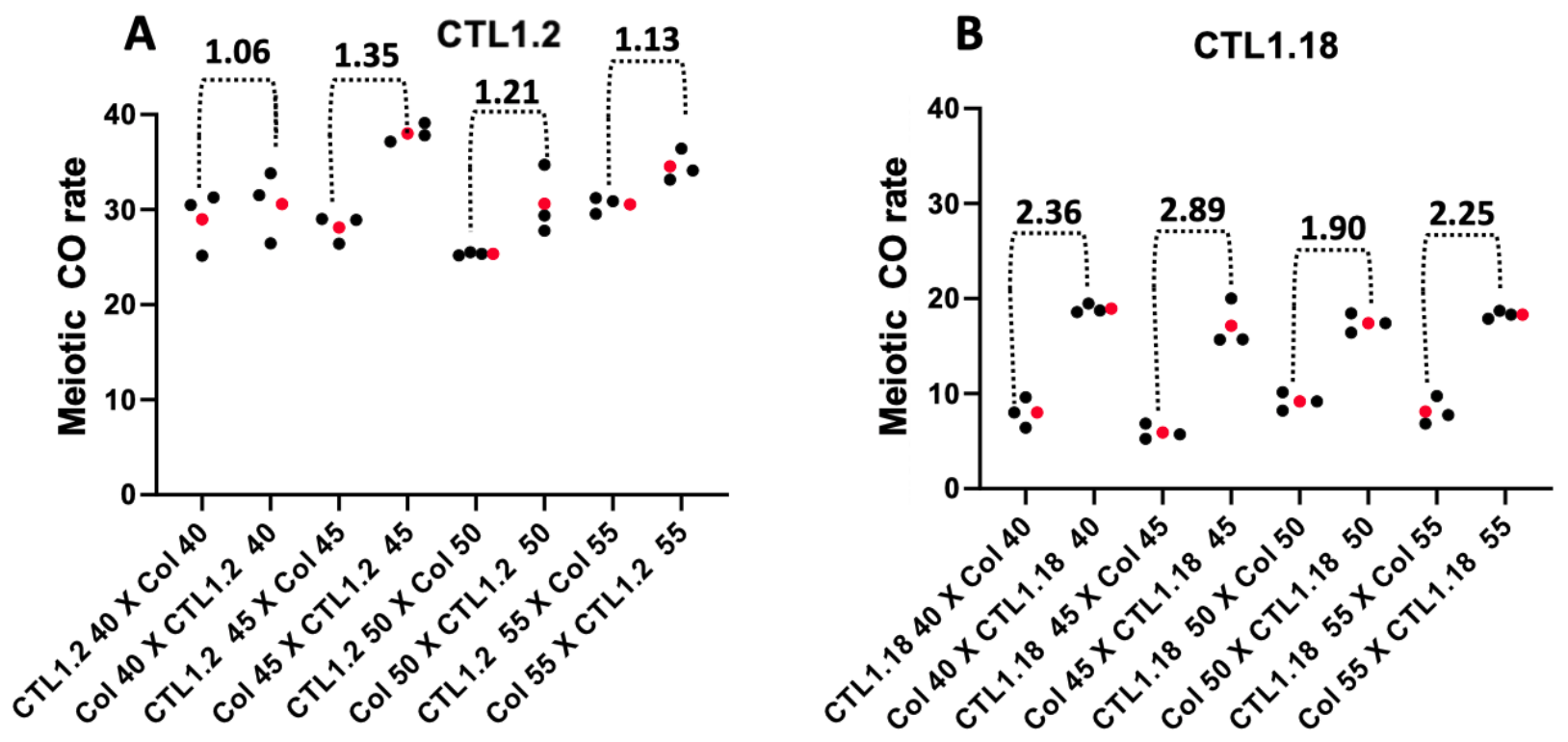

C

CTL2.4

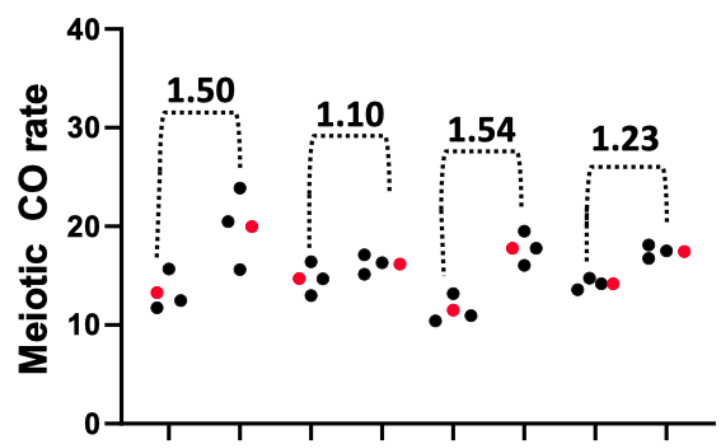

231

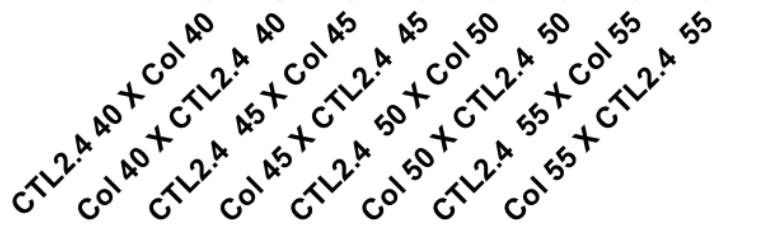

D

CTL4.7

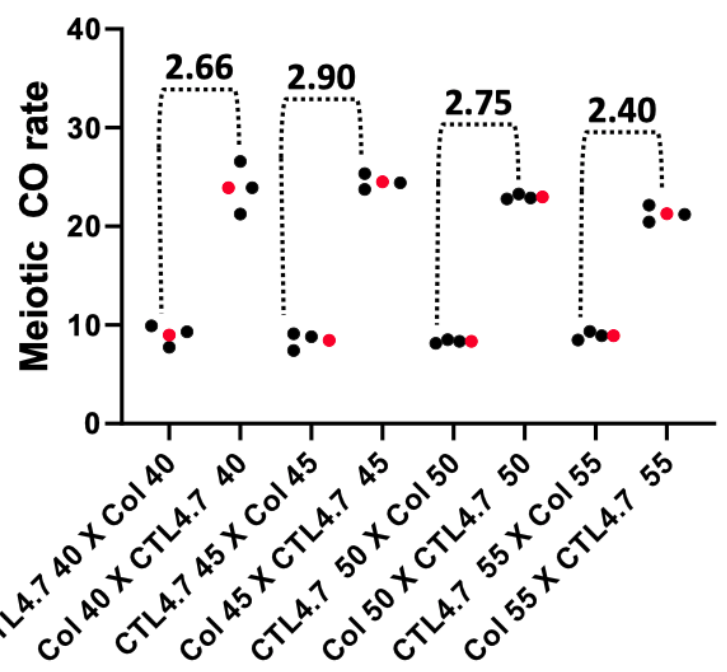



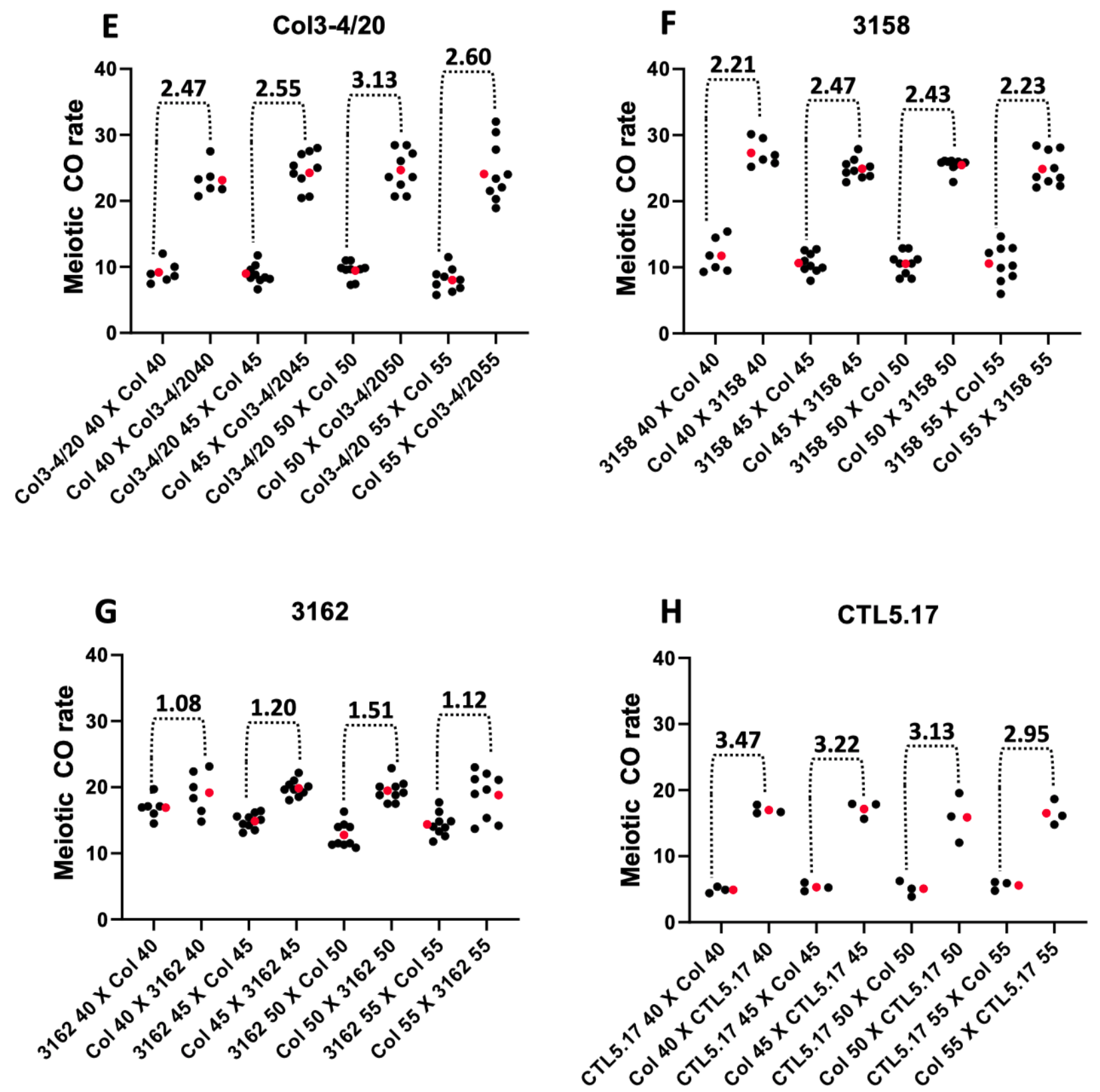

234 Figure 3 (A-H) Parental age did not affect CO rates or sex ratios: Reciprocal crosses between detector lines CTL1.2, CTL1.18, CTL2.4, CTL4.7, Col3-4/20, 3158, 3162, CTL5.17 and Columbia plants (of 40, 45, 50 and 55 DAS). Each dotted bracket spans two clusters of

237 datapoints (comprising the full set of datapoints for one age category); the first cluster is of male $238 \mathrm{CO}$ rates, the second of female $\mathrm{CO}$ rates. The number above each bracket shows the average 
ratio of the male and female rates (i.e. mCO:fCO) for each age category. The graph represents

240 individual replicated (black dots) and mean value (red dots) of the CO rates. GLM was used for

241 detecting significant difference and $P$ values were corrected for multiple testing (Supplementary

242 Tables S1-8).

245 The average rates and thus the average heterochiasmic ratios, mCO:fCO, that we have measured 246 can also be compared with those predicted by analysis of data published previously (Table S2 in

247 Giraut et al. (2011)). Their genome-wide study reported the rates of mCO and fCO at 380 shared

248 locations across all five Arabidopsis chromosomes, demonstrating remarkable variation in both

249 rates from location to location. In Table 2 we present the measured and predicted rates and ratios

250 (all ages combined), the predicted values being based on our interval-based analysis (Table S12)

251 of the location-based data presented in their Table S2 (Giraut et al. 2011). The predicted values

252 show a good congruence, in terms of their magnitudes relative to each other, with those that we

253 measured. This point is evident when the measured and predicted ratios are ranked from high or

254 low, according to their magnitude (Table 2).

Table 2 Measured and predicted male and female CO rates and their ratios

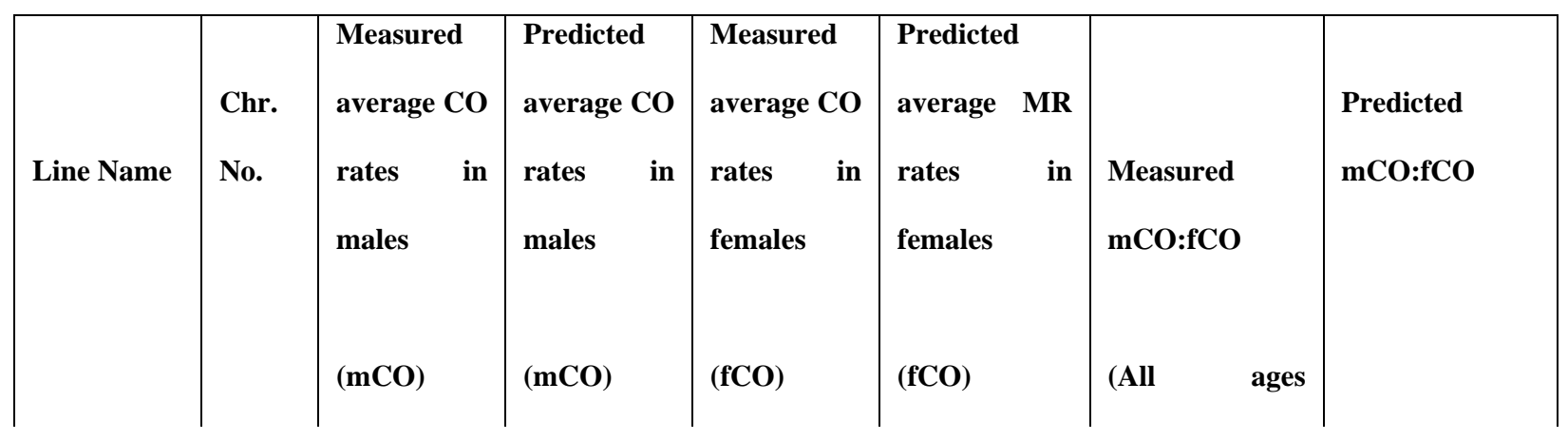




\begin{tabular}{|c|c|c|c|c|c|c|c|c|c|}
\hline & & & & & & \multicolumn{2}{|c|}{ combined) } & & \\
\hline & & & & & & Ratio & Rank & Ratio & Rank \\
\hline CTL1.2 & 1 & 33.44 & 45.26 & 28.24 & 37.59 & 1.184 & 7 & 1.20 & 8 \\
\hline $\begin{array}{l}\text { CTL1.18 } \\
\end{array}$ & 1 & 17.95 & 19.43 & 7.81 & 5.98 & 2.30 & 4 & 3.25 & 2 \\
\hline CTL2.4 & 2 & 17.84 & 17.84 & 13.41 & 13.41 & 1.33 & 5 & 2.31 & 6 \\
\hline Col3-4/20 & 3 & 24.04 & 24.04 & 8.90 & 8.90 & 2.67 & 2 & 3.49 & 1 \\
\hline 3158 & 3 & 25.66 & 25.66 & 10.88 & 10.88 & 2.33 & 3 & 3.06 & 4 \\
\hline CTL4.7 & 4 & 23.17 & 23.17 & 8.67 & 8.67 & 2.67 & 2 & 3.21 & 3 \\
\hline 3162 & 5 & 19.33 & 19.33 & 14.73 & 14.73 & 1.22 & 6 & 1.28 & 7 \\
\hline CTL5.17 & 5 & 16.62 & 15.48 & 5.21 & 5.98 & 3.20 & 1 & 2.45 & 5 \\
\hline
\end{tabular}

258 intervals studied here. The data for all age categories were combined because there was no significance

259 difference across the four ages. All the rate values are dimension-less and thus relative only to other values in

260 the same column, and to values in the corresponding measured or predicted column (i.e. column 3 values can

261 be compared to those in column 5; likewise for columns 4 and 6). The predicted rate values were based on our

262 analysis of the data provided in Giraut et al. (2011), Table S2. That data provides location-based male and

263 female CO rates for 11 to 33 points per interval. Here, for each interval, the predicted rate was calculated as the

264 average male or female CO rate across the interval, multiplied by the length of the interval (as per the values

265 shown in our Table 1). Comparison of the values in the columns labelled 'Rank' in our Table 2 show that

266 when the measured ratios are ordered by numerical magnitude, their high-low sequence is similar to that of the

267 predicted ratios. 


\section{Levels of heterochiasmy in eight intervals of Arabidopsis varied with the interval studied}

271 The ratio mCO:fCO did, however, vary significantly on an interval by interval basis (Figure 3;

272 Table 2). For example, the ratio of the average mCO:average mCO (across all four ages for any

273 one interval) varied between 1.18 (CTL1.2) and 3.20 (CTL5.17; Table 2). These two lines also

274 exhibited the most extreme values for both of the individual rates, mCO and fCO. CTL1.2,

275 which had the lowest ratio, had the highest individual rates (mCO: 33.44; fCO: 28.24); while

276 CTL5.17, which had the highest ratio, had the lowest rates (mCO: 16.62; fCO: 5.21) (Table 2).

278 The relatively higher values of the measured male and female CO rates of detector line CTL1.2

279 were also predicted by our analysis of the data from Table S2 of Giraut et al. (2011). A high CO

280 rate in an interval can be the consequence of the number and strength of its CO hotspots and/or

281 the length of the interval. In the case of CTL1.2, the high male rate was solely due to the interval

282 being two to three times longer than any of the others. According to our analysis of Table S2,

283 Giraut et al. (2011), the average male rate along this interval (as measured in the earlier study) is

284 in fact the second lowest of the eight intervals. For the relatively higher female rate of CTL1.12,

285 the interval's female hotspots also contributed: it has the highest average rate of recombination

286 of any of the intervals (calculated from Table S2, Giraut et al. (2011)).

288 The pattern of high or low sex ratios can also be predicted from our calculations of the 289 percentage overlap that intervals have with the subtelomeric and pericentromeric regions. For 290 example, the only intervals with an mCO:fCO of less than 2.0 (i.e. CTL1.2; CTL2.4; 3162; Table 
291 2), are those that have no overlap with a subtelomeric region (Table 1), which are known for

292 their concentration of male CO hotspots (Giraut et al. 2011).

293

\section{Discussion}

297 Levels of heterochiasmy in Arabidopsis likely show a wide range of intrachromosomal 298 responses to parental age

299 Our results provide new insights into a previous finding that heterochiasmy in Arabidopsis

300 decreases with age (Toyota et al. 2011). The current study, only the second to address the topic,

301 showed that in eight out of eight intervals, heterochiasmy did not change with age. However, we

302 do not believe that our results challenge the previous finding. As we will discuss below, we

303 would accept that on a genomic or chromosomal basis, the ratio mCO:fCO is likely to decrease

304 with age; but, our results, when considered together with previous work, suggest that, within any

305 given chromosome, the ratio is unlikely to decrease at many locations. That is, we propose that

306 heterochiasmy in Arabidopsis shows a wide spectrum of intrachromosomal responses to age,

307 including, at some locations, no response at all.

309 For all eight intervals studied here, distributed across all five each chromosomes, neither did 310 mCO:fCO, nor its component rates, change with age. The reliability of our ratio estimations is 311 supported by the good agreement of our results with the rates and ratios that can be calculated 312 using the values of mCO and fCO that Giraut et al. (2011) provide for multiple locations within 313 each interval. Some discrepancies might be expected given the different genetic background of 
314 the accessions used in the two studies. The relative values of the measured rates and ratios are

315 also as predicted, as accurately as can be expected, by the extent of overlap between an interval

316 and the subtelomeric and pericentromeric regions of the chromosome.

318 The likelihood that heterochiasmy shows intrachromosomal variation in its response to age is

319 supported by considering our results together with those of Li et al. (2017) and Toyota et al.

320 (2011). Li et al. (2017) studied nine intervals (none of which corresponded to any of our eight)

321 and found that $\mathrm{mCO}$ did not change with age in five of nine intervals studied, and increased in

322 two; significantly, there were two cases where, in a single chromosome, mCO changed

323 significantly with age for one interval but not another (Figure 3, Li et al. 2017). Although they

324 did not study the response of $\mathrm{fCO}$, the intrachromosomal variation in the response of $\mathrm{mCO}$

325 would also likely lead to variation in the response of mCO:fCO for the intervals studied. Further,

326 in the study of Toyota et al. (2011), there was considerable variation, across the chromosomes, in

327 the response of mCO:fCO to age. This was primarily driven by variation in the degree to which

328 fCO increased with age; this increase varied from $1 \%$ in chromosome 4 to $14 \%$ in chromosome 3

329 (analysis of Table 5; Toyota et al. 2011). Likewise, in the same study, while the response of

330 mCO to age did not change significantly when all five chromosomes were considered together, it

331 did increase by 7\% in both chromosomes 1 and 5 (analysis of Table 5; Toyota et al. 2011). It is

332 likely that these interchromosomal variations are accompanied by variation at the

333 intrachromosomal level. For example, in chromosome 1, where Toyota et al. (2011) found no

334 change in the response of $\mathrm{mCO}$, and only a $1 \%$ average change for fCO, there must be many

335 locations where the ratio $\mathrm{mCO}$ : fCO did not change with age at all - as we found for all intervals 
336 in our study. Also of note here is the study of Francis et al. (2007), which found that mCO did

337 not change in response to age.

339 From our results, together with those of the two previous studies mentioned above (Toyota et al.

340 2011; Li et al. 2017), one can propose the following: that as we consider each location along a

341 chromosome of Arabidopsis in turn, we will find that: (1) the rates of male and female meiosis

342 will change (as shown by Giraut et al. 2011), as will, for many cases, the degree of

343 heterochiasmy; and (2) the sensitivity of rates/ratio to age will change, such that the percentage

344 response to age at any one location will sit somewhere along a broad continuum of values, the

345 starting point of which is zero.

347 Which meiotic processes could be responsible for intrachromosomal variability in the response

348 of heterochiasmy to age?

350 That such a spectrum of age $\mathrm{x}$ gender responsiveness exists within a chromosome is not

351 unexpected. A chromosome is highly heterogeneous entity in many respects, and this

352 heterogeneity takes differing forms in male and female meiosis. For example, in humans,

353 synapsis initiation sites are found near the telomeres in male meiosis (Brown et al. 2005), but

354 interstitially in females (Lynn et al. 2004). Since synapsis initiation sites are also sites for CO

355 (Choi and Henderson 2015), this means that: (a) we can expect different rates of male and female

$356 \mathrm{CO}$ at two more or less defined locations within the chromosome; and (b) since each of those

357 locations will vary epigenetically in male and female meiosis (i.e. supporting or not supporting 
synapsis initiation), this opens up the possibility that each will also respond differently to the many cellular changes that accompany ageing.

360 In Arabdiopsis, there is not the same tight coupling between synapsis initiation and CO

361 (Chelysheva et al. 2007). However, we can be certain that, if we compare any pair of

362 corresponding locations in a chromosome undergoing male or female meiosis, there is a high

363 probability that their epigenetic environments will differ. A visual scan of the male and female

364 recombination landscapes of any of the five chromosomes (Figure 4, Giraut et al. 2011) shows

365 that the rates, and the ratio, mCO: $\mathrm{CO}$, vary frequently and dramatically from location to

366 location. Their analysis found that amongst 380 locations in the genome, more than half were

367 significantly hot or cold, in terms of male or female CO rates. The hotspots are thus necessarily

368 not just concentrated in one area of the chromosome: e.g. 27/40 of the male hotspots occur away

369 from the telomeres (Figure 4, Giraut et al. 2011). Given that the chromosomes involved in male

370 and female meiosis have the same sequence, a mechanistic explanation for the difference

371 between the $\mathrm{CO}$ rates at the same location must lie in their exposure to different epigenetic

372 conditions. As with the human example described in the previous paragraph, this will create the

373 potential for intrachromosomal and sex-based variation in the responses to age.

374 The mechanisms that might lead to these local chromosomal differences in mCO and fCO, or the

375 cumulative differences they bring about at the genome level, have been the focus of many studies

376 in Arabidopsis, and these have indicated a range of epigenetic mechanisms that could provide a

377 'substrate' for some or all of the intrachromosomal variation in age $\mathrm{x}$ gender responses. What is

378 interesting to consider, for our purposes, is which of the mechanisms might be both: (1) 
significantly important for the differences in male and female CO rates; and (2) highly

380 responsive to age.

382 Perhaps the strongest candidate is the degree of chromatin compaction, including -for each

383 'level' of compaction- the associated molecular players that maintain that level. In Arabidopsis,

384 female chromosomes are markedly more compact, as indexed by their much shorter 385 synaptonemal complexes (Drouaud et al. 2007); synaptonemal complex length is a known 386 indicator of chromatin compaction and a predictor of meiotic combination rate in Arabidopsis, 387 and other species (Kleckner 2006; Drouaud et al. 2007; Brachet et al. 2012; Zickler and 388 Kleckner 2015; Wang et al. 2016; Modliszewski and Copenhaver 2017). Modelling studies have 389 also indicated that sex variations in chromosomal structural axis length (which is related to 390 synaptonemal complex length) are sufficient to explain the sex variation in $\mathrm{CO}$ rates in

391 Arabidopsis (Zickler and Kleckner 2015). Control of chromatin organisation ensures that 392 chromosomes have the right length, and other structural features, that are critical to the proper 393 alignment of daughter chromatids, and thus crossing over itself (Brachet et al. 2012; Stapley et 394 al. 2017).

396 Chromatin restructuring, particularly of heterochromatin, is also well established as one of the 397 common features of cellular ageing and senescence in animals; chromatin becomes locally more 398 or less compact with age (Vaquero et al. 2003; Swanson et al. 2015). In plants, less is known, 399 but methylation patterns, which are associated with both chromatin compaction and CO rates, do 400 change with age: in Arabidopsis, for example, ageing is accompanied by DNA demethylation 401 (Ogneva et al. 2016); also, in Arabidopsis leaf senescence, heterochromatin disintegrates (Ay et 
403 the level of compaction naturally varies along the length of the chromosome, being negatively

404 correlated with gene density (Brachet et al. 2012). This, together, with the known global

405 differences between compaction levels in male and female male meiotic cells, suggests that local

406 variations in compaction level could provide the rich epigenetic substrate needed for the

407 extensive age $\mathrm{x}$ gender intrachromosomal variation indicated by the current and previous

408 findings.

\section{Conclusions}

412 Our results help, we believe, to clarify how the levels of heterochiasmy in Arabidopsis respond

413 to age. From previous work, it might be concluded that the level of disparity between rates will

414 drop universally across the genome, but we suggest that the magnitude, perhaps even the

415 direction, of the response depends on which part of the genome is sampled. This has implications

416 for other studies that look at the interactive effects of multiple factors on any given meiotic

417 response, indicating the possibility of different findings if global or local sampling approaches

418 are adopted. Our results will also be of interest to researchers who are looking for ways to bring

419 about increased CO rates in specific regions of the genome (Fernandes et al. 2018). If any given

420 approach to inducing a rate increase has been rejected in the past because it did not lead to a

421 global rate increase, the possibility nevertheless remains that the approach might induce

422 increased CO rates in a local genomic region of interest. 


\section{Author Contributions}

427 Conceived and designed the experiments: RS, AKS and RB. Performed the experiments and 428 compiled the data: RS. Analysed the data: RS, AKS, GJH, and RB. Wrote the paper: RS, AKS, 429 GJH and RB.

\section{Acknowledgements}

432 We would like to thank Avraham A. Levy (Weizmann Institute of Science, Israel) for providing 433 seeds of MR detector lines. We would like to thank the Arabidopsis Biological Resource Center 434 (The Ohio State University, USA) for providing traffic line seeds.

\section{$436 \quad$ Literature Cited}

437 Alves I., A. A. Houle, J. G. Hussin, and P. Awadalla, 2017 The impact of recombination on 438 human mutation load and disease. Philos. Trans. R. Soc. B Biol. Sci. 372: 20160465. 439 https://doi.org/10.1098/rstb.2016.0465

440 Ay N., K. Irmler, A. Fischer, R. Uhlemann, G. Reuter, et al., 2009 Epigenetic programming via

441 histone methylation at WRKY53 controls leaf senescence in Arabidopsis thaliana. Plant J. 58:

442 333-346. https://doi.org/10.1111/j.0960-7412.2009.03782.x

443 Brachet E., V. Sommermeyer, and V. Borde, 2012 Interplay between modifications of chromatin

444 and meiotic recombination hotspots. Biol. Cell 104: 51-69.

445 https://doi.org/10.1111/boc.201100113 
446 Bretz F., T. Hothorn, and P. Westfall, 2016 Multiple comparisons using R. Chapman and 447 Hall/CRC.

448 Brown P. W., L. Judis, E. R. Chan, S. Schwartz, A. Seftel, et al., 2005 Meiotic synapsis proceeds

449 from a limited number of subtelomeric sites in the human male. Am. J. Hum. Genet. 77: 556450566.

451 Charlesworth D., and X. Vekemans, 2005 How and when did Arabidopsis thaliana become 452 highly self-fertilising. BioEssays 27: 472-476. https://doi.org/10.1002/bies.20231

453 Chelysheva L., G. Gendrot, D. Vezon, M.-P. Doutriaux, R. Mercier, et al., 2007 Zip4/Spo22 is 454 required for Class I CO formation but not for synapsis completion in Arabidopsis thaliana. PLOS 455 Genet. 3: e83. https://doi.org/10.1371/journal.pgen.0030083

456 Chiang T., R. M. Schultz, and M. A. Lampson, 2012 Meiotic origins of maternal age-related 457 aneuploidy1. Biol. Reprod. 86. https://doi.org/10.1095/biolreprod.111.094367

458 Choi K., and I. R. Henderson, 2015 Meiotic recombination hotspots - a comparative view. Plant 459 J. 83: 52-61. https://doi.org/10.1111/tpj.12870

460 Drouaud J., V. Zanni, and D. Brunel, 2007 Sex-specific crossover distributions and variations in 461 interference level along Arabidopsis thaliana chromosome 4. PLoS Genet. 3: 12.

462 Fernandes J. B., M. Séguéla-Arnaud, C. Larchevêque, A. H. Lloyd, and R. Mercier, 2018 463 Unleashing meiotic crossovers in hybrid plants. Proc. Natl. Acad. Sci. 115: 2431. 464 https://doi.org/10.1073/pnas.1713078114 
465 Francis K. E., S. Y. Lam, B. D. Harrison, A. L. Bey, L. E. Berchowitz, et al., 2007 Pollen tetrad-

466 based visual assay for meiotic recombination in Arabidopsis. Proc. Natl. Acad. Sci. 104: 3913-

467 3918. https://doi.org/10.1073/pnas.0608936104

468 Gabriel K. R., 1969 Simultaneous test procedures--some theory of multiple comparisons. Ann.

469 Math. Stat. 224-250.

470 Giraut L., M. Falque, J. Drouaud, L. Pereira, O. C. Martin, et al., 2011 Genome-wide crossover

471 distribution in Arabidopsis thaliana meiosis reveals sex-specific patterns along chromosomes,

472 (M. Lichten, Ed.). PLoS Genet. 7: e1002354. https://doi.org/10.1371/journal.pgen.1002354

473 Halldorsson B. V., M. T. Hardarson, B. Kehr, U. Styrkarsdottir, A. Gylfason, et al., 2016 The

474 rate of meiotic gene conversion varies by sex and age. Nat. Genet. 48: 1377-1384.

475 https://doi.org/10.1038/ng.3669

476 Hayman D. L., and P. A. Parsons, 1962 The effect of temperature, age and an inversion on

477 recombination values and interference in the X-chromosome of Drosophila melanogaster.

478 Genetica 32: 74-88. https://doi.org/10.1007/BF01816087

479 Hussin J., M.-H. Roy-Gagnon, R. Gendron, G. Andelfinger, and P. Awadalla, 2011 Age-

480 dependent recombination rates in human pedigrees, (G. McVean, Ed.). PLoS Genet. 7:

481 e1002251. https://doi.org/10.1371/journal.pgen.1002251

482 John A., K. Vinayan, and J. Varghese, 2016 Achiasmy: Male fruit flies are not ready to mix.

483 Front. Cell Dev. Biol. 4. https://doi.org/10.3389/fcell.2016.00075 
484 Kleckner N., 2006 Chiasma formation: chromatin/axis interplay and the role(s) of the 485 synaptonemal complex. Chromosoma 115: 175.

486 Lenormand T., 2003 The evolution of sex dimorphism in recombination. Genetics 163: 811-822.

487 Lenormand T., and J. Dutheil, 2005 Recombination Difference between sexes: A role for haploid 488 selection. PLoS Biol. 3: e63. https://doi.org/10.1371/journal.pbio.0030063

489 Li F., N. De Storme, and D. Geelen, 2017 Dynamics of male meiotic recombination frequency 490 during plant development using Fluorescent Tagged Lines in Arabidopsis thaliana. Sci. Rep. 7: 491 42535. https://doi.org/10.1038/srep42535

492 López E., M. Pradillo, C. Oliver, C. Romero, N. Cuñado, et al., 2012 Looking for natural 493 variation in chiasma frequency in Arabidopsis thaliana. J. Exp. Bot. 63: 887-894. 494 https://doi.org/10.1093/jxb/err319

495 Lynn A., T. Ashley, and T. Hassold, 2004 Variation in human meiotic recombination. Annu Rev 496 Genomics Hum Genet 5: 317-349.

497 Martin H. C., R. Christ, J. G. Hussin, J. O’Connell, S. Gordon, et al., 2015 Multicohort analysis 498 of the maternal age effect on recombination. Nat. Commun. 6: 7846. 499 https://doi.org/10.1038/ncomms8846

500 McKee B. D., R. Yan, and J.-H. Tsai, 2012 Meiosis in male Drosophila. Spermatogenesis 2: 501 167-184. https://doi.org/10.4161/spmg.21800 
502 Melamed-Bessudo C., E. Yehuda, A. R. Stuitje, and A. A. Levy, 2005 A new seed-based assay

503 for meiotic recombination in Arabidopsis thaliana: Meiotic recombination in Arabidopsis

504 thaliana. Plant J. 43: 458-466. https://doi.org/10.1111/j.1365-313X.2005.02466.x

505 Modliszewski J. L., and G. P. Copenhaver, 2017 Meiotic recombination gets stressed out: CO

506 frequency is plastic under pressure. Curr. Opin. Plant Biol. 36: 95-102.

507 https://doi.org/10.1016/j.pbi.2016.11.019

508 Nagaoka S. I., T. J. Hassold, and P. A. Hunt, 2012 Human aneuploidy: mechanisms and new 509 insights into an age-old problem. Nat. Rev. Genet. Lond. 13: 493-504.

510 http://dx.doi.org.ezproxy.uws.edu.au/10.1038/nrg3245

511 Nelder J. A., and R. W. Wedderburn, 1972 Generalized linear models. J. R. Stat. Soc. Ser. Gen. $512 \quad 135: 370-384$.

513 Ogneva Z. V., A. S. Dubrovina, and K. V. Kiselev, 2016 Age-associated alterations in DNA 514 methylation and expression of methyltransferase and demethylase genes in Arabidopsis thaliana.

515 Biol. Plant. 60: 628-634. https://doi.org/10.1007/s10535-016-0638-y

516 Ritz K. R., M. A. F. Noor, and N. D. Singh, 2017 Variation in recombination rate: Adaptive or 517 not? Trends Genet. 33: 364-374. https://doi.org/10.1016/j.tig.2017.03.003

518 Saini R., A. K. Singh, S. Dhanapal, T. H. Saeed, G. J. Hyde, et al., 2017 Brief temperature stress

519 during reproductive stages alters meiotic recombination and somatic mutation rates in the 520 progeny of Arabidopsis. BMC Plant Biol. 17: 103. https://doi.org/10.1186/s12870-017-1051-1 
522 Satomura K., N. Osada, and T. Endo, 2019 Achiasmy and sex chromosome evolution. Ecol.

523 Genet. Genomics 13: 100046. https://doi.org/10.1016/j.egg.2019.100046

524 Singh A. K., T. Bashir, C. Sailer, V. Gurumoorthy, A. M. Ramakrishnan, et al., 2015 Parental

525 age affects somatic mutation rates in the progeny of flowering plants. Plant Physiol. 168: 247-

526 257. https://doi.org/10.1104/pp.15.00291

527 Stapley J., P. G. D. Feulner, S. E. Johnston, A. W. Santure, and C. M. Smadja, 2017 Variation in

528 recombination frequency and distribution across eukaryotes: patterns and processes. Philos.

529 Trans. R. Soc. B Biol. Sci. 372: 20160455. https://doi.org/10.1098/rstb.2016.0455

530 Swanson E. C., L. M. Rapkin, D. P. Bazett-Jones, and J. B. Lawrence, 2015 Unfolding the story

531 of chromatin organization in senescent cells. Nucleus 6: 254-260.

532 https://doi.org/10.1080/19491034.2015.1057670

533 Toyota M., K. Matsuda, T. Kakutani, M. Terao Morita, and M. Tasaka, 2011 Developmental

534 changes in crossover frequency in Arabidopsis: Sex and age dependence of crossover frequency.

535 Plant J. 65: 589-599. https://doi.org/10.1111/j.1365-313X.2010.04440.x

536 Vaquero A., A. Loyola, and D. Reinberg, 2003 The constantly changing face of chromatin. Sci.

537 Aging Knowl. Environ. 2003: 4.

538 Wang Z., B. Shen, J. Jiang, J. Li, and L. Ma, 2016 Effect of sex, age and genetics on crossover

539 interference in cattle. Sci. Rep. 6: 37698. https://doi.org/10.1038/srep37698

540 Wu G., G. Rossidivito, T. Hu, Y. Berlyand, and R. S. Poethig, 2015 Traffic lines: new tools for

541 genetic analysis in Arabidopsis thaliana. Genetics 200: 35-45. 
bioRxiv preprint doi: https://doi.org/10.1101/2020.02.06.938183. this version posted February 7,2020 . The copyright holder for this preprint (which was not certified by peer review) is the author/funder, who has granted bioRxiv a license to display the preprint in perpetuity. It is made available under aCC-BY-NC-ND 4.0 International license.

542 Zickler D., and N. Kleckner, 2015 Recombination, pairing, and synapsis of homologs during

543 meiosis. Cold Spring Harb. Perspect. Biol. 7. https://doi.org/10.1101/cshperspect.a016626

544 